\title{
BMJ Open Herbal medications for surgical patients: a systematic review protocol
}

To cite: Arruda APN, Ayala AP, Lopes LC, et al. Herbal medications for surgical patients: a systematic review protocol. BMJ Open 2017;7:e014290. doi:10.1136/ bmjopen-2016-014290

- Prepublication history for this paper is available online. To view these files please visit the journal online (http://dx.doi. org/10.1136/bmjopen-2016014290).

Received 14 September 2016 Revised 28 February 2017 Accepted 20 March 2017

For numbered affiliations see end of article.

Correspondence to Dr Ana Paula Nappi Arruda; ana_nappi@yahoo.com

\author{
Ana Paula Nappi Arruda, ${ }^{1}$ Ana Patricia Ayala, ${ }^{2}$ Luciane C Lopes, ${ }^{3,4}$ \\ Cristiane C Bergamaschi, ${ }^{3}$ Caio Guimarães, ${ }^{3}$ Mariana Del Grossi, ${ }^{3}$ \\ Leonardo A R Righesso, ${ }^{5}$ Arnav Agarwal, ${ }^{6,7}$ Regina El Dib ${ }^{8,9,10}$
}

\begin{abstract}
Introduction Postoperative nausea and vomiting (PONV) affect approximately $80 \%$ of surgical patients and is associated with increased length of hospital stay and systemic costs. Preoperative and postoperative pain, anxiety and depression are also commonly reported. Recent evidence regarding their safety and effectiveness has not been synthesised. The aim of this systematic review is to evaluate the efficacy and safety of herbal medications for the treatment and prevention of anxiety, depression, pain and PONV in patients undergoing laparoscopic, obstetrical/gynaecological and cardiovascular surgical procedures.
\end{abstract}

Methods and analysis The following electronic databases will be searched up to 1 October 2016 without language or publication status restrictions: CENTRAL, MEDLINE, EMBASE, CINAHL, Web of Science and LILACS. Randomised clinical trials enrolling adult surgical patients undergoing laparoscopic, obstetrical/ gynaecological and cardiovascular surgeries and managed with herbal medication versus a control group (placebo, no intervention or active control) prophylactically or therapeutically will be considered eligible. Outcomes of interest will include the following: anxiety, depression, pain, nausea and vomiting. A team of reviewers will complete title and abstract screening and full-text screening for identified hits independently and in duplicate. Data extraction, risk of bias assessments and evaluation of the overall quality of evidence for each relevant outcome reported will be conducted independently and in duplicate using the Grading of Recommendations Assessment Development and Evaluation classification system. Dichotomous data will be summarised as risk ratios; continuous data will be summarised as standard average differences with $95 \% \mathrm{Cls}$.

Ethics and dissemination This is one of the first efforts to systematically summarise existing evidence evaluating the use of herbal medications in laparoscopic, obstetrical/ gynaecological and cardiovascular surgical patients. The findings of this review will be disseminated through peerreviewed publications and conference presentations.

Systematic review registration PROSPERO CRD42016042838.

\section{INTRODUCTION}

Postoperative nausea and vomiting (PONV) and pain account for over half of reported symptoms by surgical patients. ${ }^{1}$ Defined as

\section{Strengths and limitations of this study}

This systematic review will provide an objective comprehensive and systematic assessment of the use of herbal medications for surgical patients who undergo laparoscopic, gynaecological/obstetrical and cardiac surgeries.

- The results of this systematic review may assist clinicians and health professionals make clinical decisions regarding symptom prevention and management, and may guide researchers in terms of additional questions to be addressed in relation to herbal medications with this patient population.

- While systematic and robust in its methods, this systematic review is limited primarily to the peerreviewed literature addressing herbal medications for the patient population of interest, and may not capture evidence from other sources.

nausea and/or vomiting occurring within 24 hours after surgery, reported PONV prevalences among surgical patients ranged from $25 \%$ to $30 \%$ in a number of studies, and have been reported as high as $80 \% .^{23}$ In addition to decreased quality of life, PONV has also been associated with increased hospital length of stay and systemic costs. ${ }^{4}$ While recommendations for pharmacological prophylaxis and treatment for PONV exist, these medications may be associated with notable side effects. ${ }^{5}$

Depression and anxiety are also very frequent worldwide in terms of perioperative symptoms for patients undergoing surgery, and have been associated with prolonged durations to recovery. ${ }^{67}$ Reported prevalences of anxiety have been reported to be as high as $80 \%$ in the perioperative period, ${ }^{89}$ and has been reported to be higher among those with chronic medical conditions relative to the general population. ${ }^{10}$ Depression and anxiety disorders have been associated with increased rates of readmission, ${ }^{11}$ morbidity ${ }^{12}$ and mortality ${ }^{13}$ in surgical patients.

Evidence from the USA suggests that $70 \%$ to $80 \%$ of the 23 million people who undergo surgical procedures annually experience 
moderate to severe pain. ${ }^{14}$ Another study reported a postoperative pain prevalence of $52.5 \%$ in the first 24 hours and $41.1 \%$ on the second postoperative day for hospitalised surgical patients, with the most common type of pain reported by patients being musculoskeletal $(54 \%){ }^{15}$ Generally, pain decreases over time but may persist for days or even months postoperatively. ${ }^{16}$ Postoperative pain may complicate recovery and delay discharge of patients as well. ${ }^{17}$

Conventional medications are the general treatment for this set of symptoms. Premedication with anxiolytic and sedative drugs may reduce preoperative anxiety. ${ }^{18}$ However, the role of anxiolytic premedication for surgical patients remains unclear and postoperative side effects may result from routine premedication. ${ }^{19}$ Recently, new generations of antiemetic and shorter-acting anaesthetic drugs have been used in PONV. ${ }^{20}$ Opioid agonists are the current mainstay of pain treatment after surgery, but opioid therapy is severely limited by side effects at effective doses. ${ }^{21}$ Preoperative cognitive behavioural therapy (CBT) has been associated with less postday surgery pain and a lower risk of chronic postoperative pain. ${ }^{22}$ Postoperative CBT has also been associated with decreased postoperative depression rates relative to conventional medications. ${ }^{23}$

Use of herbal medications by surgical patients is quite common worldwide for a number of these indications as well, although geographic variability exists. A study of hospitalised patients in a public medical centre in Israel found that $44 \%$ reported using herbal remedies in the last year; 89 different remedies were reportedly used. ${ }^{24}$ In comparison, the estimated prevalence of herbal medicine use for patients undergoing surgery in the USA has been reported to range from $32 \%$ to $51 \% .^{25}$ Eighty-five per cent of the Brazilian population has been reported to use medicines involving plants or plant-based preparations as part of their healthcare ${ }^{26}$ Reported prevalence rates for herbal medicine use in the European countries range from $5.9 \%$ to $48.3 \%$ across the UK, Germany, Turkey, Switzerland, Sweden, Norway, Denmark, Italy, Israel, Finland and Spain. ${ }^{27}$

While herbal medications have been associated with positive effects on postoperative pain, anxiety and PONV, ${ }^{28-30}$ they have been associated with side effects of their own. In addition, there may also be concerns regarding interactions with conventional medications and associated perioperative adverse events such as bleeding, cardiovascular instability, coagulopathy, excessive somnolence, photosensitivity and endocrine and electrolyte disturbances. ${ }^{31-37}$ Despite growing knowledge about herbal medications and drug interactions, most of these concerns have arisen based on theoretical data rather than clinical evidence from surgical patients. ${ }^{38}$

The American Society of Anaesthesiology recommends discontinuing herbal remedies consumption 2 weeks prior to surgery. ${ }^{39}$ Nevertheless, a recent study showed that only around $23 \%$ of preoperative surgical patients discontinue their herbal medication regimens prior to surgery. ${ }^{40}$

No recent systematic reviews evaluating herbal medications in patients undergoing surgical procedures for perioperative and postoperative symptom control were identified. As such, we plan to undertake a systematic review summarising the efficacy and safety of herbal medications for the treatment and prevention of anxiety, depression, pain and PONV in patients undergoing laparoscopic, obstetrical/gynaecological and cardiovascular surgical procedures.

\section{METHODS AND ANALYSIS \\ Standards}

The Cochrane Handbook for Intervention Reviews ${ }^{41}$ will guide our choice of methods. This review will adhere to the Preferred Reporting Items for Systematic Reviews and Meta-Analyses Statement. ${ }^{42}$

\section{Protocol and registration}

This review protocol is registered in the International Prospective Register of Systematic Reviews (PROSPERO CRD42016042838).

\section{Search methods for primary studies \\ Electronic searches}

We will search the following electronic databases without language and publication status restrictions, up to 1 October 2016: Cochrane Central Register of Controlled Trials (Cochrane CENTRAL), Ovid MEDLINE, Ovid Embase, LILACS, ISI Web of Science, EBSCO CINAHL and clinicaltrials.gov. Search terms describing laparoscopic, obstetrical/gynaecological and cardiovascular surgeries and herbal medication interventions will be combined. The search strategy will be designed with the assistance of a trained librarian.

\section{Search strategy}

We will use the following MeSH terms, with associated keywords: (1) intervention (phytotherapy, herb therapy, herbal therapy, plant extracts, herbal medicine, herbalism, medicinal plants, pharmaceutical plants, pharmaceutical plant, healing plants, healing plant, medicinal herbs, medicinal herb); (2) condition (laparoscopies, peritoneoscopy, peritoneoscopies, celioscopy, celioscopies, laparoscopic surgical procedures, laparoscopic surgical procedure, laparoscopic surgery, laparoscopic surgeries, cardiac surgical procedure, cardiac surgical procedures, heart surgical procedures, heart surgical procedure, cardiovascular surgical procedure, cardiovascular surgical procedures, gynaecological surgical procedure, gynaecologic surgical procedures, gynaecological surgeries, gynaecological surgery, gynaecologic surgery, gynaecologic surgeries, obstetric surgical procedure, obstetric surgical procedures, obstetrical surgical procedures, obstetrical surgical procedure, obstetric surgeries, obstetric surgery, obstetrical surgery, obstetrical surgeries). The search strategy will be adapted 
Table 1 Search strategy for Ovid MEDLINE, designed as of 2 September 2016

\begin{tabular}{|c|c|c|}
\hline No & Searches & Results \\
\hline 1 & $\begin{array}{l}\text { gynecology/ obstetrics/ thoracic surgery/ } \\
\text { Minimally Invasive Surgical Procedures/ }\end{array}$ & 61687 \\
\hline 2 & laparoscopy/ hand-assisted laparoscopy/ & 69622 \\
\hline 3 & $\begin{array}{l}\text { thoracic surgical procedures/ exp cardiac } \\
\text { surgical procedures/ }\end{array}$ & 195024 \\
\hline 4 & $\begin{array}{l}\text { expGynecologic/obstetric Surgical } \\
\text { Procedures/ }\end{array}$ & 72904 \\
\hline 5 & Cholecystectomy, Laparoscopic/ & 10733 \\
\hline 6 & $\begin{array}{l}\text { ((gynecolog* or cardiac or cardio* or } \\
\text { thoracic or heart or coronary or obstetric* } \\
\text { or gynae* or laparoscop* or OBGYN or }^{*}{\text { uter } \text { or vaginal or cervical }^{*} \text { or ovarian*) }}^{\star} \text { adj5 (surger* or operation* or operate*)). } \\
\text { tw,kf. }\end{array}$ & 153069 \\
\hline 7 & Herbal Medicine/ & 1629 \\
\hline 8 & 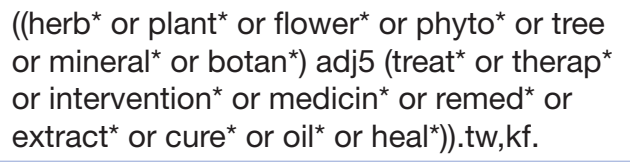 & 101339 \\
\hline 9 & (herbalism or botany or herbology).tw,kf. & 1255 \\
\hline 10 & Phytotherapy/ & 33568 \\
\hline 11 & (phyto-therap* or phytotherap*).tw,kf. & 1680 \\
\hline 12 & $\begin{array}{l}\text { exp Plant Preparations/pd, tu, ad, } \\
\text { st [Pharmacology, Therapeutic Use, } \\
\text { Administration \& Dosage, Standards] }\end{array}$ & 103896 \\
\hline 13 & /1-6 [Surgery] & 457564 \\
\hline 14 & /7-12 [Herbal medicine] & 194482 \\
\hline 15 & 13 and 14 & 1296 \\
\hline 16 & adult.mp. or middle aged.sh. or age:.tw. & 7608507 \\
\hline 17 & 15 and 16 & 470 \\
\hline
\end{tabular}

for each of the aforementioned electronic databases (see table 1 for the search strategy adapted for Ovid MEDLINE).

\section{Searching other resources}

In addition to an electronic database search, we will manually search the reference lists of every study deemed eligible to identify additional trials which may be includable; any potentially eligible studies will be screened in duplicate. Furthermore, coauthors of all eligible trials and/or pharmaceutical companies leading the conduct of eligible trials will be contacted for additional data and information regarding any additional trials which may be includable.

\section{Eligibility criteria}

\section{Inclusion criteria}

\section{Patients}

Adults ( $\geq 18$ years of age) undergoing laparoscopic, obstetrical/gynaecological and/or cardiac surgeries.

\section{Interventions}

Any herbal medicines from any of the following plant preparations (whole, powder, extract, crude drug, standardised mixture, drug extract ratio and solvent) will be compared against conventional treatment, placebo, no intervention, other type of complementary and alternative therapy (eg, acupuncture, homeopathy) or another herbal medication. We will consider the following routes of administration: oral (eg, dropping pills, aqueous decocts), topical and intravenous. We will only consider herbal medications preoperatively administrated.

\section{Study designs}

Randomised controlled trials (RCTs) and quasi-RCTs.

\section{Exclusion criteria}

Patients

Studies where the majority of participants are HIV-positive or transplant patients will not be considered eligible for inclusion.

\section{Interventions}

Studies involving combination herbal medication regimens as interventions and/or combination pharmacological medications as control arms will not be considered eligible for inclusion.

\section{Measure outcomes}

We will include studies that report any of the following outcomes:

\section{Primary outcomes}

- Anxiety (Spilberger Anxiety Inventory-IStateTrait Anxiety Inventory (STAI) and other validated instruments)

- Depression (Depression Scale-Hospital Anxiety and Depression Scale (HADS-D) and other validated instruments)

- PONV (visual analogue scale (VAS) and other validated instruments)

- Overall pain (VAS and other validated instruments)

Secondary outcomes

- Adverse events-primarily withdrawals and serious adverse events (eg, death, life-threatening, hospitalisation, disability or permanent damage)

- Number of patients reporting adverse events (as defined above)

- Quality of life (Short Form-36 and other validated instruments)

- Satisfaction with herbal medications

- Need for rescue medication

- Duration of symptoms (intervention costs with descriptive analysis)

\section{Screening, extraction and risk of bias}

Three pairs of reviewers will independently screen all titles and abstracts identified by the literature search. Full-text articles for potentially eligible studies will be obtained and screened independently by reviewer pairs 
using the same eligibility criteria as with the title and abstract screening.

Once a final set of eligible studies has been identified, reviewer pairs will independently extract data for the following variables from each study using a prestandardised data extraction form: characteristics of the study design, participants, interventions, outcomes event rates (for aforementioned primary and secondary outcomes) and duration of follow-up.

Reviewers will independently assess risk of bias by using a modified version of the Cochrane Collaboration's tool. The tool includes nine domains: adequacy of sequence generation, allocation sequence concealment, blinding of participants and caregivers, blinding of data collectors, blinding for outcome assessment, blinding of data analysts, incomplete outcome data, selective outcome reporting and the presence of other potential sources of bias not accounted for in the previously cited domains. ${ }^{434}$ For incomplete outcome data, loss to follow-up of less than $10 \%$ and a difference of less than $5 \%$ in missing data in intervention and control groups is considered low risk of bias.

Consensus for both stages of screening, data extraction and risk of bias assessments will be established by discussion and adjudication by a third reviewer as necessary.

To measure agreement between reviewers, we will use kappa statistics. Kappa values between 0.40 and 0.59 are considered to demonstrate fair agreement, while values between 0.60 and 0.80 demonstrate good agreement and values greater than or equal to 0.75 demonstrate excellent agreement. ${ }^{45}$

\section{Confidence in pooled estimates of effect}

The reviewers will use the Grading of Recommendations Assessment Development and Evaluation (GRADE) methodology to rate the quality of evidence for each outcome. Quality ratings will be assigned as high, moderate, low or very low. ${ }^{46}$ Detailed GRADE guidance will be used to assess overall risk of bias, ${ }^{47}$ imprecision, ${ }^{48}$ inconsistency, ${ }^{49}$ indirectness ${ }^{50}$ and publication bias. ${ }^{51}$ Consensus will be established by discussion and adjudication by a third reviewer as necessary, and final results will be summarised in an evidence profile.

\section{Data synthesis}

We will calculate pooled risk ratios (RRs) for dichotomous outcomes and standardised mean differences (SMD) for continuous variables with the associated 95\% CIs using random effects models with the Mantel-Haenszel statistical method. Absolute effects and 95\% CI will be calculated by multiplying pooled RRs and $95 \%$ CI by baseline risk estimates derived from the largest included RCTs in the meta-analysis.

We will address variability in results across studies by using $\mathrm{I}^{2}$ statistic and the $\mathrm{p}$ value obtained from the Cochran $\chi^{2}$ test. Our primary analyses will be based on eligible patients who have reported outcomes for each study (complete case analysis). We will assess publication bias through visual inspection of funnel plots for outcomes addressed in 10 or more studies. We will use Review Manager (RevMan) V.5.3 (Nordic Cochrane Centre, Cochrane) for all analyses. ${ }^{52}$

We will calculate the SMD with a sensitivity analysis in cases where studies report the same construct using different measurement instruments. SMDs show the intervention effect in SD units, rather than the original units of measurement, and depend on the size of the effect (the difference between means) and the SD of the outcomes (the inherent variability among participants). We will use anchor-based minimally important difference (MID) to convert the SMD into an OR and risk difference. ${ }^{53}$

To address missing participant data for dichotomous outcomes $^{54}$ and continuous outcomes, ${ }^{55}$ we will use newly developed approaches. These approaches will be only applied to outcomes which show a significant treatment effect and report sufficient missing participant data to potentially introduce clinically important bias. Thresholds for important missing participant data will be determined on an outcome-by-outcome basis.

If there are sufficient studies available, we will offer a subgroup analysis for doses (lower vs higher dose) and risk of bias (lower vs higher risk of bias). We will construct summary tables and provide a narrative synthesis if the meta-analysis is not appropriate due to excessive heterogeneity in populations, interventions, comparators, outcomes or methodologies.

\section{Summarising evidence}

We will follow the recommendation by the GRADE Working Group, presenting cumulative findings in evidence profiles. ${ }^{5156}$ These evidence profiles will provide succinct, easily understandable summaries of quality of evidence and magnitude of effects. We will use a software programme, GRADEpro (http://ims.cochrane. org/gradepro) to build these evidence profiles. The following seven elements will be included: (1) a list of up to seven important outcomes, including both outcomes of benefit and harm; (2) a measure of the typical burden of these outcomes (eg, control group, estimated baseline risk); (3) a measure of the difference between risks with and without intervention; (4) the relative magnitude of effect; (5) numbers of participants and studies addressing these outcomes, as well as follow-up time; (6) a rating of the overall confidence in the estimate of effect for each outcome; and (7) additional comments, which will include the MID if available.

\section{Ethics and dissemination}

Ethical approval is not required for this systematic review protocol because it does not involve the gathering or presentation of any individual-level patient data and, as such, does not involve any privacy concerns.

Findings will be disseminated in the form of manuscripts submitted to peer-reviewed journals and presentations at research conferences with a relevant readership/audience. 


\section{DISCUSSION}

Our review will evaluate the available evidence for herbal medications for adult surgical patients, provide estimates of the effectiveness of treatments and their associated harms and evaluate the quality of the evidence in a rigorous and consistent manner using the GRADE approach. $^{57}$

This work addresses an important gap in summarising available evidence regarding a potential management strategy for the prophylaxis and treatment of anxiety, depression, pain and PONV. Our findings may assist clinicians and health professionals make clinical decisions regarding symptom prevention and management, and may guide researchers in terms of additional questions to be addressed in relation to herbal medications with this patient population.

\section{Author affiliations}

${ }^{1}$ Department of Surgery and Orthopedics, Botucatu Medical School, UNESPUniversidade Estadual Paulista, Botucatu, São Paulo, Brazil

${ }^{2}$ Instruction \& Faculty Liaison Librarian, Gerstein Science Information Centre, University of Toronto Libraries, University of Toronto, Toronto, Ontario, Canada ${ }^{3}$ Ciências Biológicas e da Saúde, University of Sorocaba, Sorocaba, São Paulo, Brazil ${ }^{4}$ Faculdade de Ciências Farmacêuticas, Universidade Estadual Paulista, Araraquara, São Paulo, Brazil

${ }^{5}$ Department of Oral \& Maxillofacial Surgery, University Medical Center Mainz, Mainz, Renânia, Germany

${ }^{6}$ Department of Health Research Methods, Evidence, and Impact, McMaster University, Hamilton, Ontario, Canada

${ }^{7}$ School of Medicine, University of Toronto, Toronto, Ontario, Canada

${ }^{8}$ Department of Anesthesiology, Botucatu Medical School, UNESP_Universidade

Estadual Paulista, Botucatu, São Paulo, Brazil

${ }^{9}$ Institute of Urology, McMaster University, St. Joseph's Healthcare, Hamilton, Ontario, Canada

${ }^{10}$ Department of Biosciences and Oral Diagnosis, Institute of Science and Technology, UNESP—Universidade Estadual Paulista, São José dos Campos, São Paulo, Brazil

Contributors APNA is the guarantor, led the writing of the manuscript and will participate in data extraction. RED and LCL are the project managers, coinvestigators, contributed to the writing and revision of the protocol and will participate in data extraction. APA is the Trial Search Coordinator responsible for the search strategy. CCB is coinvestigator, helped to revise the protocol and will participate in data extraction. CG, MDG, LARR and AA contributed to the writing and revision of the manuscript and will participate in data extraction. All authors read and approved the final manuscript.

Funding RED was supported by Brazilian Research Council (CNPq) scholarship grant number (CNPq 310953/2015-4). CNPQ is not involved in any other aspect of the project, such as the design of the project's protocol and analysis plan.

Competing interests None declared.

Provenance and peer review Not commissioned; externally peer reviewed.

Open Access This is an Open Access article distributed in accordance with the Creative Commons Attribution Non Commercial (CC BY-NC 4.0) license, which permits others to distribute, remix, adapt, build upon this work non-commercially, and license their derivative works on different terms, provided the original work is properly cited and the use is non-commercial. See: http://creativecommons.org/ licenses/by-nc/4.0/

(C) Article author(s) (or their employer(s) unless otherwise stated in the text of the article) 2017. All rights reserved. No commercial use is permitted unless otherwise expressly granted.

\section{REFERENCES}

1. Kable AK, Gibberd RW, Spigelman AD. Adverse events in surgical patients in Australia. Int J Qual Health Care 2002;14:269-76.

2. Farhadi K, Choubsaz M, Setayeshi K, et al. The effectiveness of dry-cupping in preventing post-operative nausea and vomiting by P6 acupoint stimulation: a randomized controlled trial. Medicine 2016;95:e4770.

3. Youssef N, Orlov D, Alie T, et al. What epidural opioid results in the best analgesia outcomes and fewest side effects after surgery?: a meta-analysis of randomized controlled trials. Anesth Analg 2014;119:965-77.

4. Palazzo MG, Strunin L. Anaesthesia and emesis. I: Etiology. Can Anaesth Soc J 1984;31:178-87.

5. Gan TJ, Diemunsch P, Habib AS, et al. Consensus guidelines for the management of postoperative nausea and vomiting. Anesth Analg 2014;118:85-113.

6. Underwood MJ, Firmin RK, Jehu D. Aspects of psychological and social morbidity in patients awaiting coronary artery bypass grafting. Br Heart J 1993;69:382-4.

7. Marcolino José Álvaro Marques, Suzuki FM, Alli LAC, et al. Medida $\mathrm{Da}$ ansiedade e da depressão em pacientes no pré-operatório. estudo comparativo. Rev Bras Anestesiol 2007;57:157-66.

8. Kil HK, Kim WO, Chung WY, et al. Preoperative anxiety and pain sensitivity are independent predictors of propofol and sevoflurane requirements in general anaesthesia. Br J Anaesth 2012;108:119-25.

9. Shoar S, Naderan M, Aghajani M, et al. Prevalence and determinants of depression and anxiety symptoms in surgical patients. Oman Med J 2016;31:176-81.

10. Yohannes AM, Willgoss TG, Baldwin RC, et al. Depression and anxiety in chronic heart failure and chronic obstructive pulmonary disease: prevalence, relevance, clinical implications and management principles. Int J Geriatr Psychiatry 2010;25:1209-21.

11. Daratha KB, Barbosa-Leiker C, H Burley M, et al. Co-occurring mood disorders among hospitalized patients and risk for subsequent medical hospitalization. Gen Hosp Psychiatry 2012;34:500-5.

12. Gasse C, Laursen TM, Baune BT. Major depression and firsttime hospitalization with ischemic heart disease, cardiac procedures and mortality in the general population: a retrospective danish population-based cohort study. Eur J Prev Cardiol 2014;21:532-40.

13. Fan VS, Ramsey SD, Giardino ND, et al. Sex, depression, and risk of hospitalization and mortality in chronic obstructive pulmonary disease. Arch Intern Med 2007;167:2345-53.

14. Svensson I, Sjöström B, Haljamäe H. Assessment of pain experiences after elective surgery. $J$ Pain Symptom Manage 2000;20:193-201.

15. Boisseau N, Rabary O, Padovani B, et al. Improvement of 'dynamic analgesia' does not decrease atelectasis after thoracotomy. $\mathrm{Br} \mathrm{J}$ Anaesth 2001;87:564-9.

16. Brattwall M, Warrén Stomberg M, Rawal N, et al. Patients' assessment of 4-week recovery after ambulatory surgery. Acta Anaesthesiol Scand 2011;55:92-8.

17. Campagna S, Antonielli D'Oulx MD, Paradiso R, et al. Postoperative pain, an unmet problem in day or overnight Italian surgery patients: a prospective study. Pain Res Manag 2016;2016:1-8.

18. lizawa A, Oshima T, Kasuya $\mathrm{Y}$, et al. Oral tandospirone and clonidine provide similar relief of preoperative anxiety. Can $J$ Anaesth 2004;51:668-71.

19. $\mathrm{Ng}$ EH, Miao B, Ho PC. Anxiolytic premedication reduces preoperative anxiety and pain during oocyte retrieval. A randomized double-blinded placebo-controlled trial. Hum Reprod 2002:17:1233-8.

20. Papadimitriou L, Pourgezi T, Petropoulos G, et al. Tropisetron or ondansetron for the prevention of post-operative nausea and vomiting (PONV). Eur J Anaesthesiol 1999;16:736.

21. Dolin SJ, Cashman JN, Bland JM. Effectiveness of acute postoperative pain management: I. Evidence from published data. $\mathrm{Br}$ $J$ Anaesth 2002;89:409-23.

22. Burns JW, Moric M. Psychosocial factors appear to predict postoperative pain: interesting, but how can such information be used to reduce risk? Tech Reg Anesth Pain Management 2011;15:90-9.

23. Freedland KE, Skala JA, Carney RM, et al. Treatment of depression after coronary artery bypass surgery: a randomized controlled trial. Arch Gen Psychiatry 2009;66:387-96.

24. Levy I, Attias S, Ben-Arye E, et al. Adverse events associated with interactions with dietary and herbal supplements among inpatients. Br J Clin Pharmacol 2017;83:836-45.

25. Kaye AD, Clarke RC, Sabar R, et al. Herbal medicines: current trends in anesthesiology practice-a hospital survey. $\mathrm{J}$ Clin Anesth 2000;12:468-71. 
26. BRASIL. Política Nacional de Plantas Medicinais e Fitoterápicos. Série B Textos Básicos de Saúde 2006. http://bvsms.saude.gov.br/ bvs/publicacoes/-politica_nacional_fitoterapicos.pdf

27. Eardley S, Bishop FL, Prescott P, et al. A systematic literature review of complementary and alternative medicine prevalence in EU. Forsch Komplementmed 2012;19(Suppl 2):18-28.

28. Gharabagy PM, Zamany P, Delazar A, et al. Efficacy of Eremostachys laciniata herbal extract on mitigation of pain after hysterectomy surgery. Pak J Biol Sci 2013;16:891-4.

29. Nanthakomon T, Pongrojpaw D. The efficacy of ginger in prevention of postoperative nausea and vomiting after major gynecologic surgery. J Med Assoc Thai 2006;89(Suppl 4):S130-6.

30. Akhlaghi M, Shabanian G, Rafieian-Kopaei M, et al. Citrus aurantium blossom and preoperative anxiety. Rev Bras Anestesiol 2011;61:702-12.

31. Ang-Lee MK, Moss J, Yuan CS. Herbal medicines and perioperative care. JAMA 2001;286:208-16.

32. Norred CL, Finlayson CA. Hemorrhage after the preoperative use of complementary and alternative medicines. Aana J 2000;68:217-20.

33. Tachjian A, Maria V, Jahangir A. Use of herbal products and potential interactions in patients with cardiovascular diseases. J Am Coll Cardiol 2010;55:515-25.

34. Hodges PJ, Kam PC. The peri-operative implications of herbal medicines. Anaesthesia 2002;57:889-99.

35. Cotterill JA. Severe phototoxic reaction to laser treatment in a patient taking St John's Wort. J Cosmet Laser Ther 2001;3:159-60.

36. Rose KD, Croissant PD, Parliament CF, et al. Spontaneous spinal epidural hematoma with associated platelet dysfunction from excessive garlic ingestion: a case report. Neurosurgery 1990;26:880-2.

37. Almeida JC, Grimsley EW. Coma from the health food store: interaction between kava and alprazolam. Ann Intern Med 1996;125:940-1.

38. Levy I, Attias S, Ben-Arye E, et al. Perioperative Risks of Dietary and Herbal Supplements. World J Surg 2017;41:927-34.

39. American Society of Anesthesiologists. What you should know about your patients' use of herbal medicines. [update 2003, cited 12 Feb 2017]. http://www.wehealny.org/services/BI_Anesthesiology/herbPatient.pdf

40. Franco Ruiz S, González Maldonado P. Dietary supplements and the anesthesiologist: research results and state of the art. Rev Colomb Anesthesiol 2014;42:90-9.

41. The Cochrane Collaboration. In: Higgins JPT, Green S, eds. Cochrane Handbook for Systematic Reviews of Interventions Version 5.1.0, 2011. http://handbook.cochrane.org/ (accessed aug 2016).

42. Moher D, Liberati A, Tetzlaff J, et al. Preferred reporting items for systematic reviews and meta-analyses: the PRISMA statement. BMJ 2009;339:b2535.
43. Guyatt GH, Busse JW. Modification of Cochrane Tool to assess risk of bias in randomized trials. http://distillercer.com/resources/ (accessed aug 2016).

44. Guyatt GH, Oxman AD, Vist GE, et al. GRADE: an emerging consensus on rating quality of evidence and strength of recommendations. BMJ 2008;336:924-6.

45. Orwin RG. Evaluating coding decisions. In: Cooper $\mathrm{H}$, Hedges LV, eds. The handbook of research synthesis. New York: Russell Sage Foundation, 1994:555-62.

46. Guyatt GH, Oxman AD, Vist GE, et al. GRADE: an emerging consensus on rating quality of evidence and strength of recommendations. BMJ 2008;336:924-6.

47. Guyatt $\mathrm{GH}$, Oxman AD, Vist G, et al. GRADE guidelines: 4. Rating the quality of evidence--study limitations (risk of bias). J Clin Epidemiol 2011;64:407-15.

48. Guyatt $\mathrm{GH}$, Oxman AD, Kunz R, et al. GRADE guidelines 6. Rating the quality of evidence--imprecision. J Clin Epidemiol 2011;64:1283-93.

49. Guyatt GH, Oxman AD, Kunz R, et al. GRADE guidelines: 7 . Rating the quality of evidence--inconsistency. J Clin Epidemiol 2011;64:1294-302

50. Guyatt GH, Oxman AD, Kunz R, et al. GRADE guidelines: 8 . Rating the quality of evidence--indirectness. J Clin Epidemiol 2011;64:1303-10.

51. Guyatt GH, Oxman AD, Montori V, et al. GRADE guidelines: 5 . Rating the quality of evidence--publication Bias. J Clin Epidemiol 2011;64:1277-82.

52. The Nordic Cochrane Centre, The Cochrane Collaboration. Review Manager (RevMan). 5.3. Copenhagen: The Nordic Cochrane Centre, The Cochrane Collaboration, 2011.

53. Busse JW, Bartlett SJ, Dougados M, et al. Optimal strategies for reporting pain in clinical trials and systematic reviews: recommendations from an OMERACT 12 Workshop. J Rheumatol 2015;42:1962-70.

54. Akl EA, Johnston BC, Alonso-Coello P, et al. Addressing dichotomous data for participants excluded from trial analysis: a guide for systematic reviewers. PLoS One 2013;8:e57132.

55. Ebrahim S, Johnston BC, Akl EA, et al. Addressing continuous data measured with different instruments for participants excluded from trial analysis: a guide for systematic reviewers. J Clin Epidemiol 2014;67:560-70.

56. Guyatt $\mathrm{GH}$, Thorlund K, Oxman AD, et al. GRADE guidelines: 13. Preparing summary of findings tables and evidence profilescontinuous outcomes. J Clin Epidemiol 2013;66:173-83.

57. Guyatt $\mathrm{GH}$, Oxman $A D$, Kunz R, et al. Going from evidence to recommendations. BMJ 2008;336:1049-51. 